\title{
Dynamics of coherences in the interacting double-dot Aharonov-Bohm interferometer: Exact numerical simulations
}

\author{
Salil Bedkihal and Dvira Segal \\ Chemical Physics Theory Group, Department of Chemistry, \\ University of Toronto, Toronto, Ontario M5S 3H6, Canada
}

\begin{abstract}
We study the real time dynamics of electron coherence in a double quantum dot two-terminal AharonovBohm geometry, taking into account repulsion effects between the dots' electrons. The system is simulated by extending a numerically exact path integral method, suitable for treating transport and dissipation in biased impurity models [Phys. Rev. B 82, 205323 (2010)]. Numerical simulations at finite interaction strength are supported by master equation calculations in two other limits: assuming non-interacting electrons, and working in the Coulomb blockade regime. Focusing on the intrinsic coherence dynamics between the double-dot states, we find that its temporal characteristics are preserved under weak-to-intermediate inter-dot Coulomb interaction. In contrast, in the Coulomb blockade limit, a master equation calculation predicts coherence dynamics and a steady-state value which notably deviate from the finite interaction case.
\end{abstract}

PACS numbers: 73.63.Kv,03.65.Yz, 73.63.-b, 73.23.Hk

\section{INTRODUCTION}

Is electron transfer through quantum dot structures phase coherent, or incoherent? How do electron-electron and electron phonon interactions affect phase-coherent transport? From the other direction, what is the role of the interference phenomena on many-body effects, such as the formation of the Kondo resonance? These questions were addressed in numerous experimental and theoretical works, detecting the presence of quantum coherence in mesoscale and nanoscale objects, using Aharonov-Bohm (AB) interferometry, see for example Refs. [1-15]. In particular, oscillations in the conductance resonances of an $\mathrm{AB}$ interferometer, with either one or two quantum dots embedded in its arms, were demonstrated in Refs. [2, 3], indicating on the presence of quantum coherence. Interestingly, $\mathrm{AB}$ oscillations were also manifested in the co-tunneling regime, implying that phase coherence is involved within such processes [4].

Considering the role of electron-electron (e-e) interactions in the $\mathrm{AB}$ interferometry, a systematic analysis carried out in Ref. [5] has argued that spin flipping channels of the transferred electron, the result of e-e repulsion effects, induce dephasing. The consequence of this decohering effect was the suppression of $\mathrm{AB}$ oscillations and the appearance of an asymmetry in the resonance peaks. One should note however that this study has assumed infinitely strong e-e interactions (Coulomb blockade regime) and treated the system perturbatively in the dot-metals coupling strength. In other studies, e-e repulsion effects were totally ignored [16], incorporated using a mean-field scheme, see for example [9], or treated perturbatively using the Green function formalism [17, 18]. These studies, and other theoretical and numerical works [5, 19], have typically considered only the steady-state limit, analyzing the conductance, a linear response quantity, or the current behavior, often in the infinite large bias case [6,7].

The coherence of electron transfer processes through an AB interferometer has been typically identified and characterized via conductance oscillations in magnetic fields. However, in a double-dot $\mathrm{AB}$ structure, a device including two dots, both connected to biased metal leads, it is imperative that the relative phase between the two dot states (or charge states) should similarly convey information on electron coherence and decoherence, as this phase is tangled with the $\mathrm{AB}$ phase. In a recent work, Tu et al. [20] have analyzed this intrinsic coherence dynamics, revealing the effect of phase localization for different magnetic fluxes, by studying the real time dynamics of the two-dots reduced density matrix. This analysis, based on an exact (nonmarkovian) master equation method [21], left out e-e interaction effects all-together.

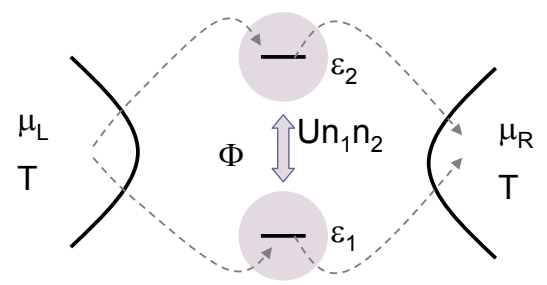

FIG. 1: Scheme of a double-dot AB interferometer. The two dots are each represented by a single electronic level. Electron repulsion energy is represented by the double arrow. The total magnetic flux is denoted by $\Phi$.

Detailed study of the dynamical role of finite electronelectron interactions on the intrinsic coherence behavior in a biased double-dot $\mathrm{AB}$ interferometer, is the focus of our work. The system includes a parallel quantum dot setup for the $A B$ interferometer, where (spinless) electrons experience an interdot repulsion effect. For a schematic representation, see Fig. 1. A unified description of the conductance behavior of this model, a steady-state property, was given in Ref. [19]. Here, we focus on the dynamics of the coherences, off diagonal elements of the double-dot reduced density matrix. Furthermore, we simulate the charge current in the system, assuming different values for the magnetic flux, at finite bias. Other effects considered are the role of finite temperature on the coherence pattern, and the behavior away from the electron-hole symmetric point, a regime not considered before in a nonperturbative calculation within the AB setup [22]. 
We follow the nonequilibrium real time dynamics of electron coherence in this subsystem-bath model (double quantum dot-metals) by performing exact numerical simulations, employing the recently developed influence functional path integral (INFPI) technique [23, 24]. This method relies on the observation that in out-of-equilibrium (and/or finite temperature) cases bath correlations have a finite range, allowing for their truncation beyond a memory time dictated by the voltagebias and the temperature. Taking advantage of this fact, an iterative-deterministic time-evolution scheme has been developed where convergence with respect to the memory length can in principle be reached. As convergence is facilitated at large bias, the method is well suited for the description of the real-time dynamics of mesoscale and nanoscale devices driven to a steady-state via interaction with biased leads. The INFPI approach is complementary to other numerically exact methods such as numerical renormalization group techniques [25, 26], real time quantum Monte Carlo simulations [27] and path integral methods [28]. It offers flexibility in defining the impurity object and the metal band structure. The results well converge at large voltage bias and/or high temperatures, as we show below.

The principles of the INFPI approach have been detailed in Refs. [23, 24], where it has been adopted for investigating dissipation effects in the nonequilibrium spin-fermion model, and the population and the current dynamics in correlated quantum dots, by investigating the single impurity Anderson model [29] and the two-level spinless Anderson dot [30]. In this paper, we further extend this approach, examining the effect of a magnetic flux on the intrinsic coherence dynamics. Our simulations show that general dynamical characteristics of the double-dot coherence are maintained upon the application of inter-dot Coulombic interactions. In particular, the characteristic timescale for reaching the steady-state limit, the dependence of the coherence on the $\mathrm{AB}$ phase factors, and the form of the temporal current, similarly evolve for systems at zero or finite inter-dot interaction, for finite bias (beyond linear response), away from the electron-hole symmetric point, at low or high temperatures. We compare our data to (analytic) results based on a master equation treatment. This method can readily handle the zero e-e interaction case and the counter case, the infinite interaction limit. Interestingly, in the latter Coulomb blockade case the coherence is expected to evolve and sustain values distinctively different from its behavior at finite interactions.

The paper is organized as follows. In Sec. II we describe the model and draw the principles of the INFPI technique. In Sec. III we present numerical results for the coherence dynamics and the charge current, analyzing the role of electronelectron interaction. Sec. IV includes analytic results based on master equations. Conclusions follow in Sec. V.

\section{MODEL AND NUMERICAL METHOD}

We focus on the symmetric $\mathrm{AB}$ setup, with a quantum dot (impurity) located at each arm of the interferometer. The dots are each connected to two metal leads (referred to as "baths" or "reservoirs"), maintained in a biased state. For simplicity, we neglect the spin degree of freedom and describe each quantum dot by a single spinless electronic level. Overall, the dots ' 1 ' and '2' are represented by the electronic levels $\epsilon_{1}$, and $\epsilon_{2}$, respectively, described by the creation operators $d_{m}^{\dagger}$, $(m=1,2)$. These levels are coupled in an AB geometry to two metal leads $(\alpha=L, R)$ with chemical potentials $\mu_{\alpha}$. For a schematic representation see Fig. 10 The total Hamiltonian, $H$, includes the following terms

$$
\begin{aligned}
& H=\epsilon_{1} n_{1}+\epsilon_{2} n_{2}+U n_{1} n_{2}+\sum_{\alpha, k} \epsilon_{k} c_{\alpha, k}^{\dagger} c_{\alpha, k} \\
& +\sum_{k, m=1,2}\left[V_{L, k, m} e^{i \phi_{m, L}} d_{m}^{\dagger} c_{L, k}+V_{R, k, m} e^{i \phi_{m, R}} c_{R, k}^{\dagger} d_{m}\right. \\
& + \text { h.c. }]
\end{aligned}
$$

Here, $c_{\alpha, k}^{\dagger}$ denotes the creation (annihilation) of an electron with momentum $k$ in the $\alpha$ lead. We assume identical leads, characterized by the same band structure. For the subsystem, $n_{m}=d_{m}^{\dagger} d_{m}$ represents the number operator for the impurity level $m, U$ is the charging energy penalty for a simultaneous occupancy at the two dots. The AB phase factors, $\phi_{m, \alpha}$, are acquired by electron waves under a magnetic field perpendicular to the device plane,

$$
\phi_{1, L}-\phi_{2, L}+\phi_{1, R}-\phi_{2, R} \equiv \phi=2 \pi \Phi / \Phi_{0} .
$$

Here $\Phi$ is the magnetic flux enclosed by the ring and $\Phi_{0}=$ $h / e$ is the flux quantum. In what follows, we adopt the following gauge, $\phi_{1, L}-\phi_{2, L}=\phi_{1, R}-\phi_{2, R}=\phi / 2$. Besides the phase factors, the coupling strength $V_{\alpha, k, m}$ are taken as real numbers. The hybridization elements are given by

$$
\Gamma_{\alpha, m, n}=\pi \sum_{k} V_{\alpha, k, m} V_{\alpha, k, n} e^{i\left(\phi_{m, \alpha}-\phi_{n, \alpha}\right)} \delta\left(\epsilon-\epsilon_{k}\right) .
$$

We assume that the couplings are identical for the two levels, $V_{\alpha, k, m}=V_{\alpha, k, n}$, and define the diagonal decay to the $\alpha$ bath

$$
\Gamma_{\alpha}=\pi \sum_{k}\left(V_{\alpha, k, m}\right)^{2} \delta\left(\epsilon-\epsilon_{k}\right) .
$$

The total diagonal decay is denoted by $\Gamma=\Gamma_{L}+\Gamma_{R}$. In practice, we take $\Gamma_{\alpha}$ to be identical at the two ends. Further, in what follows we only consider the degenerate situation with $\epsilon \equiv \epsilon_{m}$.

In the absence of magnetic fields this model is referred to as the "spinless two-level Anderson model". It has been extensively studied in the context of molecular electronics, for exploring various effects in molecular conduction [31], and in mesoscopic physics, revealing nontrivial effects such as population inversion [19, 30] and transmission phase lapses [22, 32, 33].

Using the INFPI approach, the following observables could be followed: the dots' occupation, $\left\langle n_{m}\right\rangle \equiv \operatorname{Tr}\left[d_{m}^{\dagger} d_{m} \rho\right]$, the coherence, $\sigma_{1,2} \equiv \operatorname{Tr}\left[d_{1}^{\dagger} d_{2} \rho\right]$, and the total current passing through the interferometer. The trace is performed over all degrees of freedom, metals and impurity. The charge current 
presented will be the symmetrized current, $\left\langle I_{e}\right\rangle \equiv \operatorname{Tr}\left[\hat{I}_{e} \rho\right]$, accessed by defining the operator of interest as

$$
\begin{aligned}
\hat{I}_{e} & =-\Im \sum_{k, m} V_{L, k, m} c_{L, k}^{\dagger} d_{m} e^{-i \phi_{m, L}} \\
& +\Im \sum_{k, m} V_{R, k, m} c_{R, k}^{\dagger} d_{m} e^{i \phi_{m, R}},
\end{aligned}
$$

with $\Im$ denoting the imaginary part. Within INFPI, these observables are simulated in the Heisenberg representation as we explain below, assuming an initial density matrix $\rho(0)$ describing a nonequilibrium-biased situation.

We outline now the principles of the INFPI method, allowing for the exact simulation of transport and dissipation in impurity models [23, 24]. We begin by reorganizing the Hamiltonian, Eq. (1), as $H=H_{0}+H_{1}$, identifying the nontrivial many-body interaction term as

$$
H_{1}=U\left[n_{1} n_{2}-\frac{1}{2}\left(n_{1}+n_{2}\right)\right] .
$$

$H_{0}$ contains the remaining two-body terms, redefining the dot energies as $E_{d, m}=\epsilon_{m}+U / 2$. This partitioning allows us to utilize the Hubbard-Stratonovich (HS) transformation [34], see Eq. (8) below. Formally, the dynamics of a quadratic operator, $\hat{A}$, either given in terms of the baths (metals) or impurity degrees of freedom, can be written as

$$
\langle\hat{A}(t)\rangle=\operatorname{Tr}[\rho(0) \hat{A}(t)]=\lim _{\lambda \rightarrow 0} \frac{\partial}{\partial \lambda} \operatorname{Tr}\left[\rho(0) e^{i H t} e^{\lambda \hat{A}} e^{-i H t}\right]
$$

Here $\lambda$ a real number, taken to vanish at the end of the calculation, $\rho$ is the total density matrix, and the trace is performed over both subsystem and reservoirs degrees of freedom. For simplicity, we assume that at the initial time $t=0$ the dots and the baths are decoupled, $\rho(0)=\sigma(0) \otimes \rho_{L} \otimes \rho_{R}$. The baths are prepared in a nonequilibrium biased state $\rho_{\alpha}$; the subsystem is described by the (reduced) density matrix $\sigma(0)$.

We proceed and factorize the time evolution operator, $e^{i H t}=\left(e^{i H \delta t}\right)^{N}$, further utilizing the Trotter decomposition $e^{i H \delta t} \approx\left(e^{i H_{0} \delta t / 2} e^{i H_{1} \delta t} e^{i H_{0} \delta t / 2}\right)$. The many-body term $H_{1}$ can be eliminated by introducing auxiliary Ising variables $s= \pm$ via the HS transformation [34],

$$
e^{ \pm i H_{1} \delta t}=\frac{1}{2} \sum_{s} e^{H_{ \pm}(s)} ; \quad e^{H_{ \pm}(s)} \equiv e^{-s \kappa_{ \pm}\left(n_{2}-n_{1}\right)}
$$

Here $\kappa_{ \pm}=\kappa^{\prime} \mp i \kappa^{\prime \prime}, \kappa^{\prime}=\sinh ^{-1}[\sin (\delta t U / 2)]^{1 / 2}, \kappa^{\prime \prime}=$ $\sin ^{-1}[\sin (\delta t U / 2)]^{1 / 2}$. The uniqueness of this transformation requires $U \delta t<\pi$. Incorporating the Trotter decomposition and the HS transformation into Eq. (7), the time evolution of $\hat{A}$ is dictated by

$$
\langle\hat{A}(t)\rangle=\lim _{\lambda \rightarrow 0} \frac{\partial}{\partial \lambda}\left\{\int d s_{1}^{ \pm} d s_{2}^{ \pm}, \ldots, d s_{N}^{ \pm} I\left(s_{1}^{ \pm}, s_{2}^{ \pm}, \ldots, s_{N}^{ \pm}\right)\right\}
$$

The integrand, referred to as as the "Influence Functional" (IF), is given by $(k=1, k+p=N)$

$$
I\left(s_{k}^{ \pm}, \ldots, s_{k+p}^{ \pm}\right)=\frac{1}{2^{2(p+1)}} \operatorname{Tr}\left[\rho(0) \mathcal{G}_{+}\left(s_{k+p}^{+}\right) \ldots \mathcal{G}_{+}\left(s_{k}^{+}\right) e^{i H_{0}(k-1) \delta t} e^{\lambda \hat{A}} e^{-i H_{0}(k-1) \delta t} \mathcal{G}_{-}\left(s_{k}^{-}\right) \ldots \mathcal{G}_{-}\left(s_{k+p}^{-}\right)\right]
$$

Here $\mathcal{G}_{+}\left(s_{k}^{+}\right)=\left(e^{i H_{0} \delta t / 2} e^{H_{+}\left(s_{k}^{+}\right)} e^{i H_{0} \delta t / 2}\right)$ and $\mathcal{G}_{-}=\mathcal{G}_{+}^{\dagger}$. Eq. (9) is exact in the $\delta t \rightarrow 0$ limit. Practically, it can be evaluated by noting that in standard nonequilibrium situations, even at zero temperature, bath correlations die exponentially, thus the IF in Eq. (9) can be truncated beyond a memory time $\tau_{c}=N_{s} \delta t$, corresponding to the time beyond which bath correlations may be controllably ignored [23]. Here $N_{s}$ is an integer and the correlation time $\tau_{c}$ is determined by the nonequilibrium situation, roughly $\tau_{c} \sim 1 / \Delta \mu$. This argument implies the following (non-unique) breakup [23]

$$
\begin{aligned}
& I\left(s_{1}^{ \pm}, s_{2}^{ \pm}, \ldots s_{N}^{ \pm}\right) \simeq I\left(s_{1}^{ \pm}, s_{2}^{ \pm}, \ldots, s_{N_{s}}^{ \pm}\right) I_{s}\left(s_{2}^{ \pm}, s_{3}^{ \pm}, \ldots, s_{N_{s}+1}^{ \pm}\right) \ldots \\
& \times I_{s}\left(s_{N-N_{s}+1}^{ \pm}, s_{N-N_{s}+2}^{ \pm}, \ldots, s_{N}^{ \pm}\right),
\end{aligned}
$$

where each element in the product, besides the first one, is given by a ratio between truncated IF,

$$
I_{s}\left(s_{k}, s_{k+1}, \ldots, s_{k+N_{s}-1}\right)=\frac{I\left(s_{k}^{ \pm}, s_{k+1}^{ \pm}, \ldots, s_{k+N_{s}-1}^{ \pm}\right)}{I\left(s_{k}^{ \pm}, s_{k+1}^{ \pm}, \ldots, s_{k+N_{s}-2}^{ \pm}\right)} .
$$

It is useful to define the multi-time object

$$
\begin{aligned}
& \mathcal{R}\left(s_{k+1}^{ \pm}, s_{k+2}^{ \pm}, \ldots, s_{k+N_{s}-1}^{ \pm}\right) \equiv \\
& \sum_{s_{1}^{ \pm}, s_{2}^{ \pm}, \ldots, s_{k}^{ \pm}} I\left(s_{1}^{ \pm}, s_{2}^{ \pm}, \ldots, s_{N_{s}}^{ \pm}\right) I_{s}\left(s_{2}^{ \pm}, s_{3}^{ \pm}, \ldots, s_{N_{s}+1}^{ \pm}\right) \ldots \\
& \times I_{s}\left(s_{k}^{ \pm}, s_{k+1}^{ \pm}, \ldots, s_{k+N_{s}-1}^{ \pm}\right),
\end{aligned}
$$

and time-evolve it by multiplying it with the subsequent truncated IF, then summing over the intermediate variables,

$$
\begin{aligned}
& \mathcal{R}\left(s_{k+2}^{ \pm}, s_{k+3}^{ \pm}, \ldots, s_{k+N_{s}}^{ \pm}\right)= \\
& \sum_{s_{k+1}^{ \pm}} \mathcal{R}\left(s_{k+1}^{ \pm}, s_{k+2}^{ \pm}, \ldots, s_{k+N_{s}-1}^{ \pm}\right) I_{s}\left(s_{k+1}^{ \pm}, s_{k+2}^{ \pm}, \ldots, s_{k+N_{s}}^{ \pm}\right) .
\end{aligned}
$$

Summation over the internal variables results in the time local 
expectation value,

$$
\left\langle e^{\lambda \hat{A}\left(t_{k}\right)}\right\rangle=\sum_{s_{k+2-N_{s}}^{ \pm}, \ldots, s_{k}^{ \pm}} \mathcal{R}\left(s_{k+2-N_{s}}^{ \pm}, s_{k+3-N_{s}}^{ \pm}, \ldots, s_{k}^{ \pm}\right)(15)
$$

This procedure should be repeated for several (small) values of $\lambda$. Taking the numerical derivative with respect to $\lambda$, the expectation value $\left\langle\hat{A}\left(t_{k}\right)\right\rangle$ is retrieved.

The main element in this procedure, the truncated IF [Eq. [10], is calculated using a fermionic trace formula [35],

$$
\begin{aligned}
I & =\operatorname{Tr}\left[e^{M_{1}} e^{M_{2}} \ldots e^{M_{p}}\left(\rho_{L} \otimes \rho_{R} \otimes \sigma(0)\right)\right] \\
& =\operatorname{det}\left\{\left[I_{L}-f_{L}\right] \otimes\left[I_{R}-f_{R}\right] \otimes\left[I_{S}-f_{S}\right]\right. \\
& \left.+e^{m_{1}} e^{m_{2}} \ldots e^{m_{p}}\left[f_{L} \otimes f_{R} \otimes f_{S}\right]\right\}
\end{aligned}
$$

Here, $\rho_{\alpha}$, the time-zero density matrix of the $\alpha=L, R$ fermion bath and $\sigma(0)$, the subsystem initial density matrix, are assumed to follow an exponential form. Other terms $e^{M}$, with $M$ a quadratic operator, represent further factors in Eq. (10). In the determinant, $m$ is a single-particle operator, corresponding to the quadratic operator $M=\sum_{i, j}(m)_{i, j} c_{i}^{\dagger} c_{j}$; $c_{i}^{\dagger}\left(c_{j}\right)$ are fermionic creation and annihilation operators, either related to the system or the baths. The matrices $I_{\alpha}$ and $I_{S}$ are the identity matrices for the $\alpha$ space and for the subsystem, respectively. The functions $f_{L}$ and $f_{R}$ are the bands electrons' energy distribution, $f_{\alpha}=\left[e^{\beta_{\alpha}\left(\epsilon-\mu_{\alpha}\right)}+1\right]^{-1}$, with the chemical potential $\mu_{\alpha}$ and inverse temperature $\beta_{\alpha}$.

The determinant in Eq. 16 is evaluated numerically by taking into account $L_{s}$ electronic states for each metal. This discretization implies a numerical error. However, we have found that with $L_{s} \sim 100$ states we can reach convergence in the time interval of interest. Other sources of error, elaborated and examined in Refs. [23, 24], are the Trotter error, originating from the approximate factorization of the total Hamiltonian into the non-commuting $H_{0}$ (two-body) and $H_{1}$ (manybody) terms, and the memory error, resulting from the truncation of the IF. Convergence is verified by demonstrating that results are insensitive to the time step and the memory size, once the proper memory time is accounted for.

As we show below, distinct observables may require different memory time $\tau_{c}$ for reaching convergence: The dots' occupation and the real part of the subsystem off-diagonal element, $\Re \sigma_{1,2}$, converge for $\tau_{c} \sim 1 / \Delta \mu$. In contrast, the charge current and $\Im \sigma_{1,2}$ require a memory time at least twice longer, as these quantities are sensitive to the bias drop at each contact, rather than to the overall voltage bias. It is important to note that this scaling is approximate, and the actual memory time further depends on the subsystem (dots) energetics in a complex way: First, the memory time depends on $U$ in a nontrivial manner [36]. In the absence of $U$ INFPI numerical results are exact, irrespective of the memory size used in the simulation. This can be seen from Eqs. 10) and (11), where a cancellation effect takes place leaving free propagation terms only, from $t=0$ to the current time. At infinitely large $U$ one expects again superior convergence behavior, as simultaneous occupancy is forbidden [36]. Second, the position of the dot states with respect to the left and right chemical potentials affect the convergence behavior. We generally found that when the dot states are located within the bias window a shorter memory time is required for reaching convergence, in comparison to the case where the dot energies are out-ofresonance with the bias window. This could be rationalized by noting that the decorrelation time for electrons within the bias window is short relative to the characteristic timescale of electrons occupying off-resonance states.

\section{INFPI NUMERICAL RESULTS}

We present here data for the coherence dynamics $\sigma_{1,2}(t)=$ $\left\langle d_{1}^{\dagger}(t) d_{2}(t)\right\rangle$ and the charge current $\left\langle I_{e}\right\rangle$ within the interacting double-dot $\mathrm{AB}$ interferometer. As we show below, we find that finite e-e interactions do not destroy the general characteristics of the coherence behavior, for the cases $U / \Gamma \leq 4$ considered here. We focus on the following set of parameters: The double-dot subsystem includes two degenerate states with $\epsilon \equiv \epsilon_{m}(m=1,2)$. The dynamics is studied away from the electron-hole symmetric point, $E_{d}=\epsilon+U / 2=0.2$. The metals' band structure is taken identical at the two ends, and we use leads with constant density of states and a sharp cutoff at $D= \pm 1$. The inter-dot repulsion is taken at the range $U=0-0.2$, whereas the system-bath hybridization strength (see definitions in Sec. II) is taken as $\Gamma=0.05$. As we demonstrate below, our results generally converge for $U / \Gamma \leq 4$. The bias voltage is applied in a symmetric manner, $\mu_{L}=-\mu_{R}$, and we take $\mu_{L}-\mu_{R}=\Delta \mu \sim 0.6$. The temperature is varied, where $\beta=1 / T=200$ corresponds to the low- $T$ case, and $\beta=5$ reflects a high- $T$ situation. The numerical parameters adopted are $L_{s} \sim 100$ states per bath, time step of $\delta t \sim 0.8-1.6$ and a memory time $\tau_{c} \sim 3-10$. This choice of bath states suffices for mimicking a continuous band structure [23, 24]. Also, recurrence effects are not observed before $t \Gamma \sim 10$. For simulating dynamics beyond that time larger reservoirs are constructed, as necessary. The time step was selected based on two (contrary) considerations: (i) It should be made short enough, for justifying the Trotter breakup, $\delta t U<1$. (ii) For computational reasons, it should be made long enough, to allow coverage of the system memory time with few terms, $N_{s}<8$, recalling that $\tau_{c}=\delta t N_{s}$.

Before presenting our results we explain the initial condition adopted. At time $t=0$ the double-dot levels are both empty, while the (decoupled) reservoirs are separately prepared with occupation functions obeying the Fermi-Dirac statistics at a given temperature $T$ and bias.

\section{A. Coherence dynamics at $U=0$}

We begin by presenting results for the noninteracting case, $U=0$. Figures 2 and 3 display the time evolution of the real and imaginary parts of $\sigma_{1,2}(t)$, respectively, for relatively large bias $\Delta \mu=0.6$ and at low temperature. We find that $\Re \sigma_{1,2}$ decays at a flux dependent rate after the initial rise. The imaginary part, displayed in Fig. 3, saturates with a time scale 


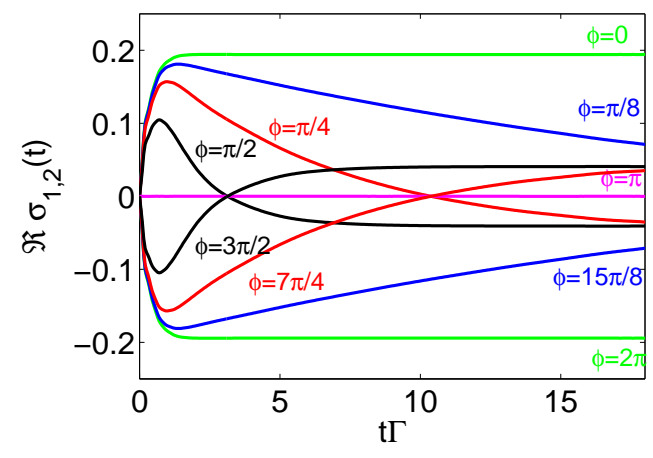

FIG. 2: Time evolution of the states coherence, in the absence of electron repulsion effects. Shown is the real part of $\sigma_{1,2}(t)$, plotted for the phases $\phi$ ranging from 0 to $2 \pi$, top to bottom. $E_{d}=0.2$, $\Gamma=0.05, U=0, \Delta \mu=0.6, \beta=200, L_{s}=240$.

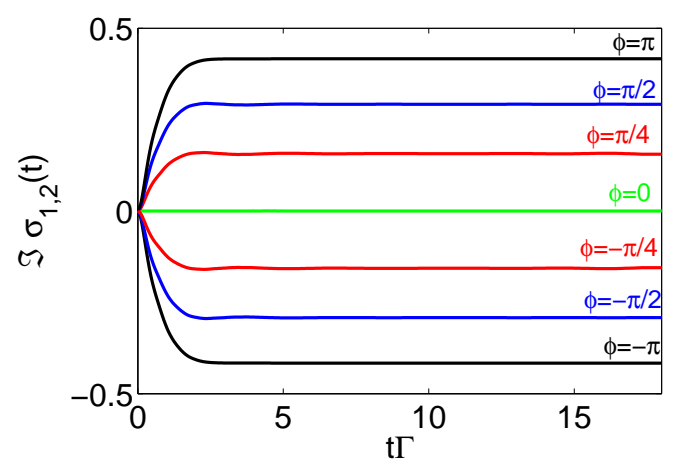

FIG. 3: Time evolution of the imaginary part of $\sigma_{1,2}(t)$, in the absence of electron repulsion effects. The phase factors $\phi$ range between $-\pi$ to $\pi$, bottom to top. Other parameters are the same as in Fig. 2]

$\Gamma$ [20]. Defining $\sigma_{1,2}(t)=\left|\sigma_{1,2}(t)\right| e^{i \varphi(t)}$, it was argued in Ref. [20] that this relative phase localizes to the values $\varphi=$ $-\pi / 2$ or $\pi / 2$ in the long time limit when $\phi \neq 2 p \pi, p$ is an integer. This localization behavior is expected only when the (degenerate) dot levels are symmetrically placed between the chemical potentials, i.e., for $\epsilon=0$. Away from this symmetric point, using $\epsilon=0.2$, Fig. 2 shows that the real part of $\sigma_{1,2}$ is finite in the asymptotic limit for any phase, besides $\pi$. It is interesting to note though that when $\phi \neq 2 p \pi, \Re \sigma_{1,2}$ still approaches a certain-fixed value, irrespective of the magnetic flux.

\section{B. Coherence and current at finite $U$}

We now investigate the role of e-e repulsion effects on the coherence behavior. Fig. 4 displays the real part of $\sigma_{1,2}(t)$ for two phases, $\phi=0$ and $\phi=\pi / 2$, and its imaginary part for $\phi=\pi$ (inset), for three values of $U$. Data for $\Im \sigma_{1,2}(t)$ at $U=0.2$ has not yet converged for the $\tau_{c}$ adopted, see text following Fig. 10. In comparison to the $U=0$ case, we find that general trends are maintained, though the long time coherences are larger in the finite $U$ case. Note our convention:

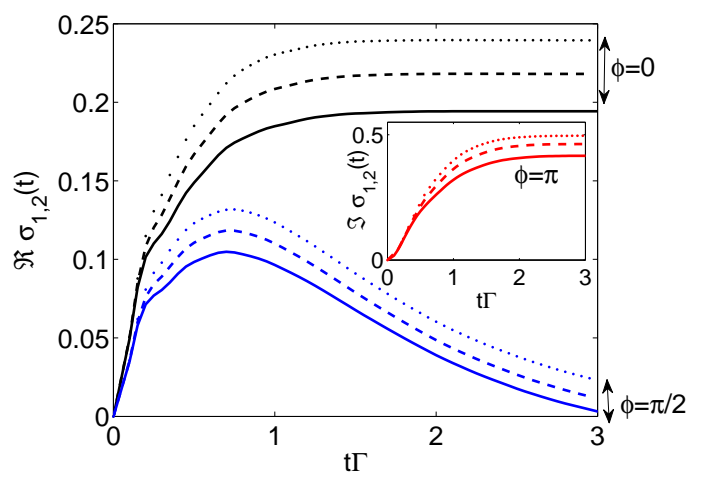

FIG. 4: Time evolution of $\sigma_{1,2}$ for $U=0$ (full line), $U=0.1$ (dashed line) and $U=0.2$ (dotted line). Main: Real part of $\sigma_{1,2}(t)$. The three top lines were simulated for $\phi=0$. The bottom lines were obtained using $\phi=\pi / 2$. The numerical parameters are $\delta t=1$, $N_{s}=6$ and $L_{s}=120$. Inset: Imaginary part of $\sigma_{1,2}(t)$ when $\phi=\pi$. Numerical parameters are $\delta t=1.6, N_{s}=6$ and $L_{s}=120$. Other parameters are $E_{d}=0.2, \Gamma=0.05, \Delta \mu=0.6$ and $\beta=200$.
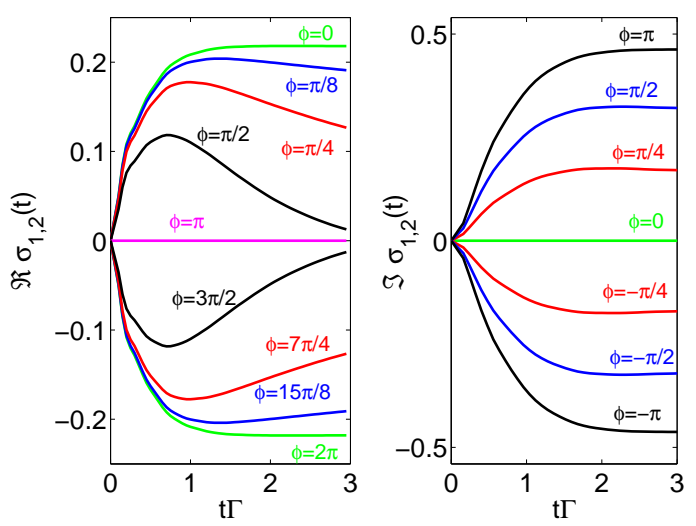

FIG. 5: Time evolution of the states coherence for $U=0.1$, for several phase factors. $E_{d}=0.2, \Gamma=0.05, \Delta \mu=0.6, \beta=200$. The real part of $\sigma_{1,2}$ was obtained with $\delta t=1$ and $N_{s}=6$; the imaginary part was simulated with $\delta t=1.6$ and $N_{s}=6$.

the parameter $E_{d}=\epsilon+U / 2$ is maintained fixed between simulations with different values of $U$. The trajectory simulated extends up to $\Gamma t=3$, where convergence is satisfactory. Different memory times were used for simulating the real part of the coherence, and its imaginary part. We adopted $\tau_{c} \sim 5$ when simulating $\Re \sigma_{1,2}$, whereas $\tau_{c} \sim 10$ was used for acquiring $\Im \sigma_{1,2}$. A more detailed discussion of convergence issues is given in Sec. III.C.

Fig. 5 presents $\sigma_{1,2}(t)$ for several phases $\phi$, at $U=0.1$. By comparing the data to the zero- $U$ case (see Figs. 2] and 3, we conclude that the symmetry of the off-diagonal elements is maintained in the presence of $U$. The general pattern of the coherence is displayed in Figs. 6 and 7 plotting the behavior of $\sigma_{1,2}$ as a function of the phase factor, at a particular time, $\Gamma t=2$, for $U=0,0.1$, and 0.2 , at different temperatures. It should be noted that by this time the real part of the coherence has not yet reached its steady-state value. We find that the 
coherence symmetry around $\phi=\pi$ (for $\Re \sigma_{1,2}$ ) or $\phi=0$ (for $\left.\Im \sigma_{1,2}\right)$ is maintained, though the absolute numbers change. Interestingly, while the effect of the temperature is significant for $\Im \sigma_{1,2}$, showing visible reduction in values at high $T$, the real part of $\sigma_{1,2}$ is only lightly affected by the temperature. The downfall of $\Im \sigma_{1,2}$ with temperature is also reflected in the behavior of the charge current, as we show next.

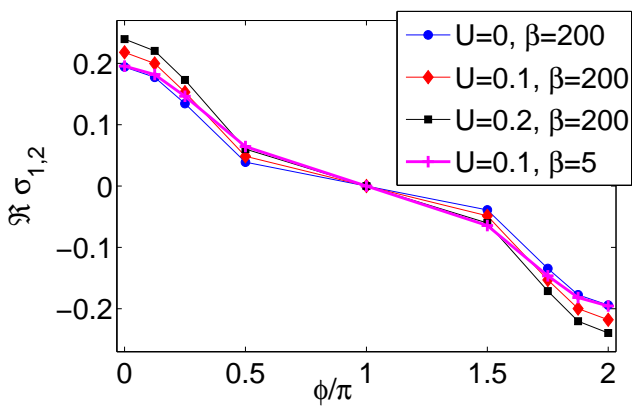

FIG. 6: Effect of finite $U$ on the coherence. $\Re \sigma_{1,2}$ is plotted as a function of the phase factor $\phi$ at a particular time, $\Gamma t=2$, for different $U$-values and temperatures. Other parameters are the same as in Fig. 5

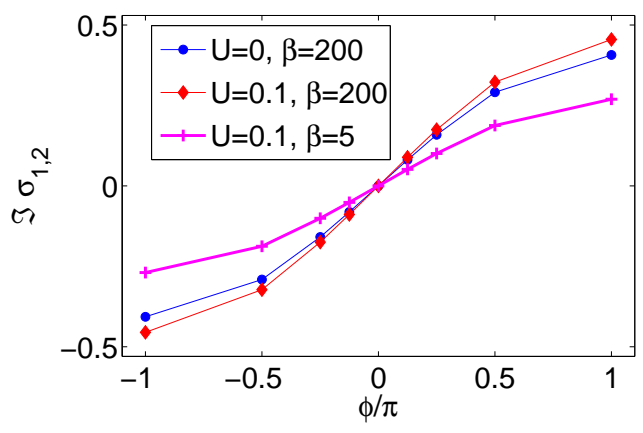

FIG. 7: Effect of finite $U$ on the coherence. $\Im \sigma_{1,2}$ is plotted as a function of $\phi$ at a particular time, $\Gamma t=2$, at finite $U$ and for different temperatures. Other parameters are the same as in Fig.5

We study the behavior of the charge current at different phases, for different e-e repulsion strengths and temperatures. Fig. 8 shows, as expected, a destructive interference pattern for electron current in the long time limit when $\phi=\pi$, irrespective of the value of $U$. This perfect destructive interference indicates that charge transport is fully coherent in this model. The temporal behavior does show however a sensitivity to the value of $U$, manifesting that systems with variable $U$ differently respond to the initial condition.

In the steady-stat limit the current scales like $\left\langle I_{e}\right\rangle \propto[1+$ $\cos (\phi)]$, for finite $U$ [5]. This relation does not hold in the short time limit. It is interesting to note that irrespective of $U$ and the phase factor the current approaches the steady-state limit on a relatively short timescale, $\Gamma t \sim 2$. At high temperatures, Fig. 8 manifests that the system is still fully coherent, while temporal oscillations are washed out. The reduction of the current at high temperatures can be attributed to the softening of the contacts' Fermi functions from the sharp step-like form at low temperatures. The electronic states at the right lead in the bias window are not fully empty any longer. Similarly, at the left lead electronic states overlapping with $E_{d}$ may be empty. Overall, this results in the reduction of the current at high $T$.

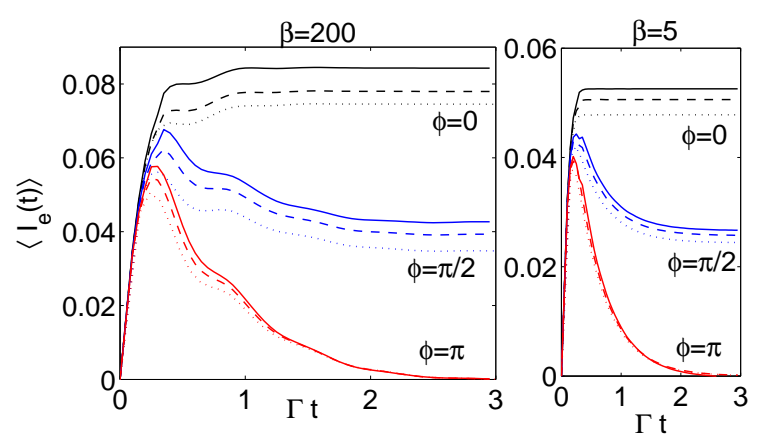

FIG. 8: Charge current through an $\mathrm{AB}$ interferometer at low temperatures, $\beta=200$ (left panel) and high temperatures $\beta=5$ (right panel) for $\phi=0, \pi / 2$, and $\pi$, top to bottom with $U=0.2$ (full line), $U=0.1$ (dashed line), $U=0$ (dotted line). Other parameters are the same as in Fig. 5. Numerical parameters are $\delta t=1, N_{s}=6$ and $L_{s}=120$.

\section{Convergence analysis}

We exemplify here the convergence behavior of the real and imaginary parts of $\sigma_{1,2}$ at low temperatures, as well as the behavior of the current. Fig. 9 demonstrates that $\Re \sigma_{1,2}$ nicely converges for $U=0.2$, for $\tau_{c} \geq 5$. The asymptotic limit is practically reached, within $\sim 1.5 \%$ error, already for $\tau_{c} \sim 1 / \Delta \mu$. We confirm that the results are insensitive to the particular time step selected (inset). We have also verified (not shown) that simulations performed with different phase factors similarly converge.

The convergence of $\Im \sigma_{1,2}$ is generally slower, as we show in Fig. 10. While $\Re \sigma_{1,2}$ converges for $\tau_{c} \gtrsim 1 / \Delta \mu$, we find that $\Im \sigma_{1,2}$ requires memory time at least twice longer for achieving convergence. For $U=0.1 \Im \sigma_{1,2}$ is converging. In contrast, at stronger interactions, $U=0.2$, the large time step adopted results in a Trotter error buildup, and the results seem to diverge around $\tau_{c} \sim 10-12$ (inset).

We also present the behavior of the charge current at different $\tau_{c}$ values, see Fig. 11. It generally converges when $\tau_{c} \sim 6$, irrespective of the phase factor (not shown), for $U / \Gamma \leq 4$, in agreement with earlier studies [24].

Overall, we conclude that we can faithfully simulate the time evolution of the coherence $\sigma_{1,2}$ and the current for $\Delta \mu=$ 0.6 and $U / \Gamma=2$. For larger $U$, the real part of $\sigma_{1,2}$, the dot occupation, and the current can be still converged [23, 24]. The simulation of $\Im \sigma_{1,2}$ requires longer $\tau_{c}$ and a shorter time step at $U / \Gamma>2$. Roughly, these observations can be rationalized noting that the dynamics of $\Re \sigma_{1,2}$ is influenced by the full potential drop, $\mu_{L}-\mu_{R}$, similarly to the dots occupation $\left\langle n_{m}\right\rangle$ [20]. In contrast, the dynamics of $\varsigma_{1,2}$ is sensitive to 
the bias drop at each contact [20], resulting in longer decorrelation times.

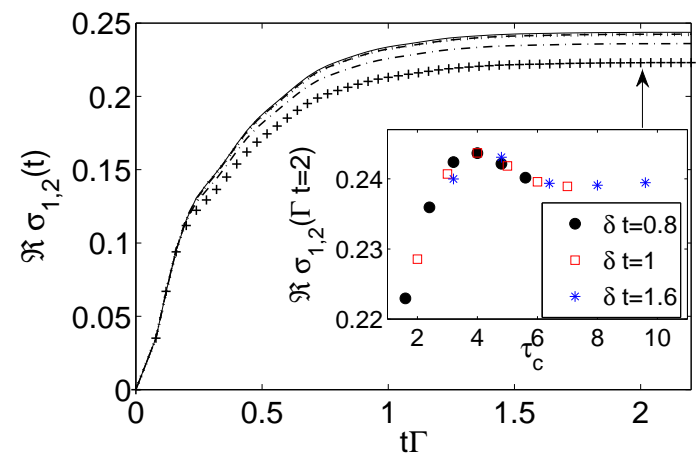

FIG. 9: Convergence behavior of $\Re \sigma_{1,2}$ for $\phi=0$ and $U=0.2$. Other physical parameters are the same as in Fig. 5 Numerical parameters are $\delta t=0.8$ and $N_{s}=2(+), N_{s}=3$ (dashed-dotted line), $N_{s}=4$ (dashed line), $N_{s}=5$ (full line) and $N_{s}=6$ (dotted line). The inset zooms on the convergence at a particular time, $\Gamma t=2$, as a function of the memory time $\tau_{c}=N_{s} \delta t$, using three different values for the time steps, $\delta t=0.8(\circ), \delta t=1(\square) \delta t=1.6(*)$.

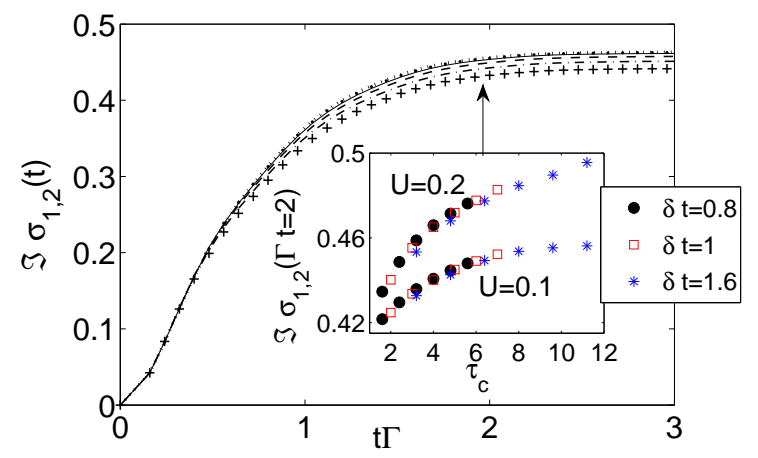

FIG. 10: Convergence behavior of $\Im \sigma_{1,2}$ for $\phi=\pi$ and $U=0.1$. Other physical parameters are the same as in Fig. 5 Numerical parameters are $\delta t=1.6$ and $N_{s}=2(+), N_{s}=3$ (dashed-dotted), $N_{s}=4$ (dashed line), $N_{s}=5$ (full line) and $N_{s}=6(\mathrm{dot}), N_{s}=7$ (dotted line). The inset presents $\varsigma_{1,2}$ at a particular time, $\Gamma t=2$, for $U=0.1$ and $U=0.2$, as a function of the memory time $\tau_{c}=$ $N_{s} \delta t$, using three different time steps, $\delta t=0.8(\circ), \delta t=1(\square)$ $\delta t=1.6(*)$.

\section{MASTER EQUATION ANALYSIS: $U=0$ AND $U=\infty$}

Rate equations for resonant transport in interacting multidot structures can be derived based on the microscopic manybody Schrödinger equation [37]. We support INFPI numerical simulations with an analytical study of the system's dynamics, based on such a master equation description. Specifically, we adopt the Bloch-type equations derived in Ref. [6], for the reduced density matrix of the double-dot system in the charge state basis $\sigma_{j, j^{\prime}}(t), j=a, b, c, d$. Here the index $j$

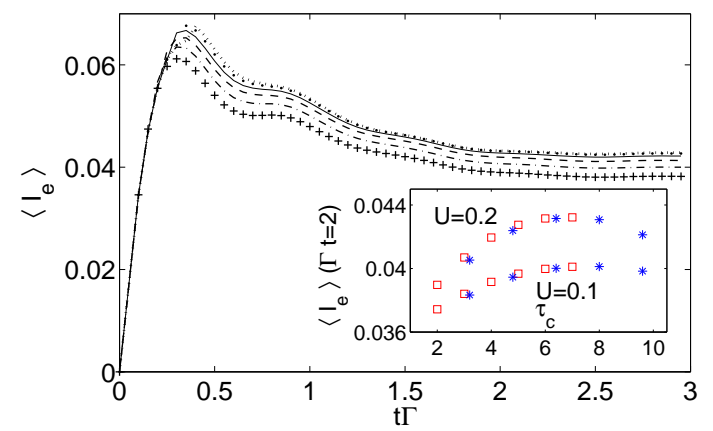

FIG. 11: Convergence behavior of the charge current, $\phi=\pi / 2$ and $U=0.2$. Other physical parameters are the same as in Fig. 5 Numerical parameters are $\delta t=1$ and $N_{s}=2(+), N_{s}=3$ (dashed-dotted), $N_{s}=4$ (dashed line), $N_{s}=5$ (full line) and $N_{s}=6$ (dot), $N_{s}=7$ (dotted line). The inset presents the data at a particular time, $\Gamma t=2$, for $U=0.1$ (bottom) and $U=0.2$ (top), as a function of the memory time $\tau_{c}=N_{s} \delta t$, using $\delta t=1(\square)$ and $\delta t=1.6(*)$.

labels the double-dot charge states in order of an empty dot $(a)$, single occupied dot, on either the "1" or " 2 " sites ( $b$ and $c$ states, respectively) and the state $(d)$, with the two dots occupied. Explicitly, $|a\rangle \leftrightarrow|00\rangle,|b\rangle \leftrightarrow|10\rangle,|c\rangle \leftrightarrow|01\rangle$, and $|d\rangle \leftrightarrow|11\rangle$. The creation and annihilation operators of the dot are related to this states by $d_{1}^{\dagger} \leftrightarrow|00\rangle\langle 01|+| 01\rangle\langle 11|$ and $d_{2}^{\dagger} \leftrightarrow|00\rangle\langle 10|+| 10\rangle\langle 11|$. Since $d_{1}^{\dagger} d_{2} \leftrightarrow|01\rangle\langle 01|$, we identify the observable of interest $\sigma_{1,2}=\operatorname{Tr}\left[\rho d_{1}^{\dagger} d_{2}\right]$ by $\sigma_{b, c}$. In the noninteracting $(U=0)$ case, the following equations hold in in the infinite bias limit [6]

$$
\begin{aligned}
\dot{\sigma}_{a, a} & =-4 \Gamma_{L} \sigma_{a, a} \\
& +2 \Gamma_{R}\left(\sigma_{b, b}+\sigma_{c, c}+\sigma_{b, c} e^{i \phi / 2}+\sigma_{c, b} e^{-i \phi / 2}\right) \\
\dot{\sigma}_{b, b} & =2 \Gamma_{L} \sigma_{a, a}-2\left(\Gamma_{R}+\Gamma_{L}\right) \sigma_{b, b}+2 \Gamma_{R} \sigma_{d, d} \\
& +\delta \Gamma^{*} e^{i \phi / 2} \sigma_{b, c}+\delta \Gamma e^{-i \phi / 2} \sigma_{c, b} \\
\dot{\sigma}_{c, c} & =2 \Gamma_{L} \sigma_{a, a}-2\left(\Gamma_{R}+\Gamma_{L}\right) \sigma_{c, c}+2 \Gamma_{R} \sigma_{d, d} \\
& +\delta \Gamma^{*} e^{i \phi / 2} \sigma_{b, c}+\delta \Gamma e^{-i \phi / 2} \sigma_{c, b} \\
\dot{\sigma}_{d, d} & =2 \Gamma_{L}\left(\sigma_{b, b}+\sigma_{c, c}-e^{-i \phi / 2} \sigma_{b, c}-e^{i \phi / 2} \sigma_{c, b}\right) \\
& -4 \Gamma_{R} \sigma_{d, d} \\
\dot{\sigma}_{b, c} & =2 \Gamma_{L} e^{i \phi / 2} \sigma_{a, a}+\delta \Gamma\left(\sigma_{b, b}+\sigma_{c, c}\right) e^{-i \phi / 2} \\
& -2 \Gamma_{R} \sigma_{d, d} e^{-i \phi / 2}-2\left(\Gamma_{L}+\Gamma_{R}\right) \sigma_{b, c} .
\end{aligned}
$$

Here $\delta \Gamma=\left(e^{i \phi} \Gamma_{L}-\Gamma_{R}\right)$. The hybridization strength, independent of the site index $m$, is defined as $\Gamma_{\alpha}=$ $\pi \sum_{k} V_{L, k, m}^{2} \delta\left(\epsilon-\epsilon_{k}\right)$. The equations are valid in the infinite bias limit, when $\left|\mu_{L}-\mu_{R}\right| \gg \Gamma$. The total probability, to occupy any of the four states, is unity, $\sum_{j=a, b, c, d} \sigma_{j, j}=1$. In the steady-state limit we demand that $d \vec{\sigma} / d t=0$, the vector $\vec{\sigma}$ includes the matrix elements $\sigma_{k, j}$ of Eq. 17, and obtain the stationary solution, valid for $\phi \neq 0$,

$$
\sigma_{b, c}(t \rightarrow \infty)=\frac{i}{2} \sin (\phi / 2)
$$

This expression holds in the symmetric setup, $\Gamma_{L}=\Gamma_{R}$, for $\phi \neq 2 \pi p, p$ is an integer. One could formally write 
$\sigma_{b, c}(t)=\left|\sigma_{b, c}(t)\right| e^{i \varphi(t)}$, noting that $\varphi$ equals $\pm \pi / 2$ in the steady-state limit. This "phase-localization" behavior was explored in Ref. [20]: The imaginary part of $\sigma_{b, c}$ depends on the magnetic phase factor, maximal for $\phi=\pi$ with the value $1 / 2$. The real part is identically zero. The results of Figs. 2 and 3 demonstrate the corresponding behavior at finite bias. There, the real part is finite, yet small, approaching a fixed value. The imaginary part slightly deviates from the prediction of Eq. (18) due to the finite bias used. One could also get hold of the characteristic rates from the dynamical equation, by diagonalizing the matrix $M$ in $d \vec{\sigma} / d t=M \vec{\sigma}$. We gather five rates, with two phase dependent rates, $\propto[1 \pm \cos (\phi / 2)]$. For small $\phi$, the smallest rate is $\propto[1-\cos (\phi / 2)]$, in agreement with [20]. It can be also proved that in this noninteracting case the steady-state current scales with $\left\langle I_{e}\right\rangle \propto[1+\cos (\phi)][6]$.

The dynamics of the coherence, attained from the master equation (17), is displayed in Fig. 12 for $\phi=\pi / 2$. In the long time limit the real part approaches zero; the imaginary part reaches $\frac{1}{2} \sin (\pi / 4)=0.354$. INFPI results at zero $U$ are also included in dotted lines. Deviations of INFPI simulations from master equation results can be traced down to the finite band used within INFPI, in comparison to the infiniteflat band assumed in the master equation approach. For finite $U$, we have found that at large bias, $\Delta \mu=2 D$, INFPI data basically overlaps with the $U=0$ case (not shown) as the system basically stands on the symmetric point.

In the Coulomb blockade regime, for $U / \Gamma \rightarrow \infty$, a strikingly different behavior is expected. Starting with the manybody Schrödinger equation, one can again derive the system's equations of motion in the large bias limit while excluding simultaneous occupancy at both dots, $\sigma_{d, d}=0$. The following equations of motion are then achieved [6, 37] $\left(\sigma_{a, a}+\sigma_{b, b}+\sigma_{c, c}=1\right)$,

$$
\begin{aligned}
\dot{\sigma}_{a, a} & =-4 \Gamma_{L} \sigma_{a, a} \\
& +2 \Gamma_{R}\left(\sigma_{b, b}+\sigma_{c, c}+\sigma_{b, c} e^{i \phi / 2}+\sigma_{c, b} e^{-i \phi / 2}\right) \\
\dot{\sigma}_{b, b} & =2 \Gamma_{L} \sigma_{a, a} \\
& -2 \Gamma_{R} \sigma_{b, b}-\Gamma_{R}\left(\sigma_{b, c} e^{i \phi / 2}+\sigma_{c, b} e^{-i \phi / 2}\right) \\
\dot{\sigma}_{c, c} & =2 \Gamma_{L} \sigma_{a, a} \\
& -2 \Gamma_{R} \sigma_{c, c}-\Gamma_{R}\left(\sigma_{b, c} e^{i \phi / 2}+\sigma_{c, b} e^{-i \phi / 2}\right) \\
\dot{\sigma}_{b, c} & =2 \Gamma_{L} e^{i \phi / 2} \sigma_{a, a} \\
& -\Gamma_{R} e^{-i \phi / 2}\left(\sigma_{b, b}+\sigma_{c, c}\right)-2 \Gamma_{R} \sigma_{b, c} .
\end{aligned}
$$

For a spatially symmetric junction, $\Gamma_{L}=\Gamma_{R}$, the steady-state solution for the coherence is

$$
\begin{aligned}
\sigma_{b, c}(t \rightarrow \infty) & =-\frac{1}{2} e^{-i \phi / 2} \\
& =-\frac{1}{2} \cos (\phi / 2)+\frac{i}{2} \sin (\phi / 2) .
\end{aligned}
$$

While the imaginary part predicted is identical to the $U=0$ case, see Eq. (18), the real part is finite and phase dependent. The Coulomb blockade dynamics is presented in Fig. 12 (dashed lines). We find that the imaginary part is weakly sensitive to the onset of $U$. In contrast, the real part significantly deviates from the $U=0$ case already at $\Gamma t \sim 1$. By analyzing the eigenvalues of the rate matrix (19), we note that phase dependent relaxation rates in the Coulomb blockade regime are the same as for noninteracting electrons, see also Fig. 12, It would be interesting to explore this evolution within the INFPI approach. However, as we are currently limited to $U / \Gamma \leq 4$ values, this would require an algorithmic improvement of the INFPI technique. We believe that such an extension could be achieved since the Coulomb blockade case should converge faster than the intermediate $U$ limit [36]. This issue will be tackled in our future work. A related switching behavior was observed in Ref. [6], where the current, finite in the noninteracting case for $\phi \neq \pi$, vanishes in the Coulomb blockade regime for any phase satisfying $\phi \neq 2 \pi p$.

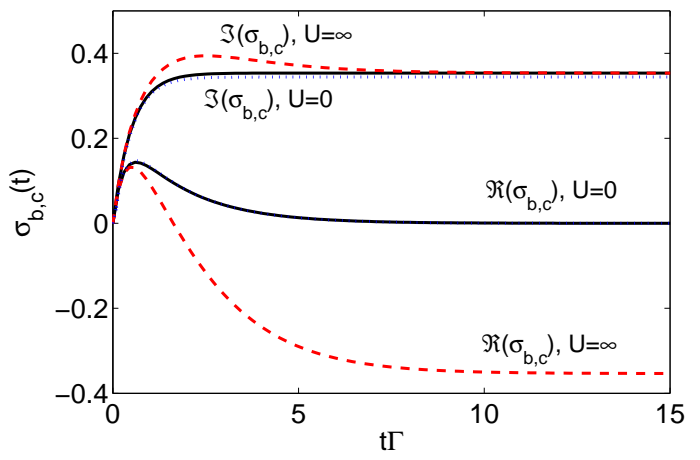

FIG. 12: Master equation analysis: Real and imaginary parts of $\sigma_{b, c}$ at $\phi=\pi / 2$, for $U=0$ and $U=\infty$, obtained by simulating Eq. 177 and Eq. 19, respectively. Results from INFPI method with $U=0$ are represented by dotted lines, practically overlapping with master equation curves.

\section{SUMMARY}

The intrinsic coherence dynamics in a double quantum dot $\mathrm{AB}$ interferometer, away from the symmetric point, was simulated using an exact numerical technique. At finite interactions, $U / \Gamma \leq 4$, at low or high temperatures, we have found that the coherence evolves similarly to the $U=0$ case, showing related characteristic timescales and long time values. Specifically, we found that for $\phi=\pi / 2$ the real part of $\sigma_{1,2}$ approaches a small number (zero at the symmetric point), while the imaginary part is larger, $\sim 0.35$. On the other hand, a master equation treatment in the Coulomb blockade regime predicts a significantly different behavior: The magnitude of $\Re \sigma_{1,2}$ and $\Im \sigma_{1,2}$ should be the same, $\sqrt{2} / 4$, for the $\phi=\pi / 2$ phase factor.

Future work will be devoted to the study of related models, including the spin degree of freedom at each dot. This model should demonstrate a decoherence process due to the intrinsic spin-flipping dephasing effect [5]. Other topics of interest are algorithmic improvement of the INFPI technique, to allow for the study of the Coulomb blockade regime. One could also add a local degree of freedom on one of the interferometer arms, e.g., a quantum point contact [38] or a vibrational mode 
[39], and observe the time evolution of the interference pattern in this "which path" experiment.

\section{Acknowledgments}

DS acknowledges support from NSERC discovery grant. The research of SB was supported by an Early Research
Award of DS. Fruitful discussions with M. Bandyopadhyay are acknowledged.
[1] G. Hackenbroich, Phys. Rep. 343, 463 (2001), and references therein.

[2] A. Yacoby, M. Heiblum, D. Mahalu, and H. Shtrikman, Phys. Rev. Lett. 74, 4047 (1995); R. Schuster, E. Buks, M. Heiblum, D. Mahalu, V. Umansky, and H. Shtrikman, Nature 385, 417 (1997); M. Avinun-Kalish, M. Heiblum, O. Zarchin, D. Mahalu, and V. Umansky, Nature 436, 529 (2005).

[3] A. W. Holleitner, C. E. Decker, H. Quin, K. Eberl, and R. H. Blick, Phys. Rev. Lett. 87, 256802 (2001).

[4] M. Sigrist,T. Ihn, K. Ensslin, D. Loss, M. Reinwald, and W. Wegscheider, Phys. Rev. Lett. 96, 036804 (2006).

[5] J. König and Y. Gefen, Phys. Rev. Lett. 86, 3855 (2001); Phys. Rev. B 65, 045316 (2002).

[6] F. Li, X.-Q. Li, W.-M. Zhang, and S. A. Gurvitz, Euro. Phys. Lett. 88, 37001 (2009).

[7] Y. Tokura, H. Nakano, and T. Kubo, New J. Phys. 9, 113 (2007).

[8] Y.-S. Liu, H. Chen, and X.-F. Yang, J. Phys.: Condens. Matt. 19, 246201 (2007).

[9] D. I. Golosov and Y. Gefen, New J. Phys. 9, 120 (2007).

[10] W. Hofstetter, J. König, and H. Schoeller, Phys. Rev. Lett. 87, 156803 (2001).

[11] D. Boese, W. Hofstetter, and H. Schoeller, Phys. Rev. B 66, 125315 (2002).

[12] Q.-F. Sun and H. Guo, Phys. Rev. B 66, 155308 (2002).

[13] J. Malecki and I. Affleck, Phys. Rev. B 82, 165426 (2010).

[14] O. Hod, E. Rabani, and R. Baer, Acc. Chem. Res. 39, 109 (2006).

[15] D. Rai, O. Hod, and A. Nitzan, J. Phys. Chem. Lett. 2, 2118 (2011).

[16] B. Kubala and J. König, Phys. Rev. B 65, 245301 (2002).

[17] D. Sztenkiel and R. Swirkowicz, J. Phys.: Condens. Matter 19 386224 (2007).

[18] Y.-S. Liu, H. Chen, and X.-F. Yang, J. Phys.: Condens. Matter 19246201 (2007).

[19] V. Kashcheyevs, A. Schiller, A. Aharony, and O. EntinWohlman, Phys. Rev. B 75, 115313 (2007).

[20] M. W.-Y. Tu, W.-M. Zhang, J. Jin, Phys. Rev. B 83, 115318 (2011).

[21] J. S. Jin, M. W. Y. Tu, W. M. Zhang, and Y. J. Yan, New J. Phys. 12, 083013 (2010).
[22] H. A. Nilsson, et al., Phys. Rev. Lett. 104, 186804 (2010); O. Karlström, J. N. Pedersen, P. Samuelsson, A. Wacker, Phys. Rev. B 83, 205412 (2011).

[23] D. Segal, A. J. Millis, and D. R. Reichman, Phys. Rev. B 82, 205323 (2010).

[24] D. Segal, A. J. Millis, and D. R. Reichman, Chem. Phys. Phys. Chem 13, 14378 (2011).

[25] R. Bulla, T. A. Costi, and T. Pruschke, Rev. Mod. Phys. 80, 395 (2008).

[26] F. B. Anders and A. Schiller, Phys. Rev. Lett. 95, 196801 (2005); Phys. Rev. B 74, 245113 (2006); F. B. Anders, Phys. Rev. Lett. 101, 066804 (2008).

[27] E. Gull, A. J. Millis, A. I. Lichtenstein, A. N. Rubtsov, M. Troyer, and P. Werner, Rev. Mod. Phys. 83, 349 (2011).

[28] S. Weiss, J. Eckel, M. Thorwart, and R. Egger, Phys. Rev. B 77, 195316 (2008); J. Eckel, F. Heidrich-Meisner, S. G. Jakobs, M. Thorwart, M. Pletyukhov, and R. Egger, New J. Phys. 12, 043042 (2010).

[29] A. C. Hewson, The Kondo Problem to Heavy Fermions, (Cambridge University Press, Cambridge, England, 1993).

[30] M. Sindel, A. Silva, Y. Oreg, and J. von Delft, Phys. Rev. B 72, 125316 (2005).

[31] M. Galperin, M. A. Ratner, and A. Nitzan, J. Phys.: Condens. Matter 19, 103201 (2007).

[32] V. Meden and F. Marquardt, Phys. Rev. Lett. 96, 146801 (2006).

[33] C. Karrasch, T. Hecht, A. Weichselbaum, J. von Delft, Y. Oreg, and V. Meden, New J. Phys. 9, 123 (2007).

[34] J. E. Hirsch, Phys. Rev. B 28, 4059 (1983).

[35] I. Klich, in "Quantum Noise in Mesoscopic Systems", edited by Yu. V. Nazarov and Ya. M. Blanter (Kluwer, 2003).

[36] G. Cohen and E. Rabani, Phys. Rev. B 84, 075150 (2011).

[37] S. A. Gurvitz and Ya. S. Prager, Phys. Rev. B 53, 15932 (1996); S. A. Gurvitz, Phys. Rev. B 57, 6602 (1998); S. A. Gurvitz, D. Mozyrsky, and G. P. Berman, Phys. Rev. B 72, 205341 (2005).

[38] I. L. Aleiner, N. S. Wingreen, and Y. Meir, Phys. Rev. Lett. 79, 3740 (1997)

[39] R. Härtle, C. Benesch, and M. Thoss, Phys. Rev. Lett. 102, 146801 (2009). 